\title{
IMMUNOSUPPRESSION IN CORNEAL TRANSPLANTATION
}

\author{
JOHN C. HILL \\ Cape Town, South Africa
}

\begin{abstract}
SUMMARY
This paper reviews the clinical post-operative management of keratoplasty and the management of corneal graft rejection. In both instances corticosteroids remain the mainstay of treatment; however, the literature shows a wide range for both route and frequency of administration. Grafts at 'high risk' require more immunosuppressive therapy, but no universally accepted definition of high risk exists and consequently different treatment regimens are difficult to compare and evaluate. Studies using univariate and multivariate survival analysis suggest that recipient corneas can be divided into low, medium and high risk depending on the number of quadrants of vascularisation (avascular, 1-2 quadrants and 3+ quadrants respectively). This wider classification would make the devising and comparing of treatment regimens more consistent. In high-risk cases, corticosteroids alone provide insufficient immunosuppression and systemic cyclosporine is needed in exceptional cases. When managing rejection episodes, a severe reaction involving the endothelium often does not respond to topical steroids alone, and systemic corticosteroids are required. Instead of oral steroids, we now prefer to use an intravenous 'pulse' of $500 \mathrm{mg}$ methylprednisolone: this is at least as effective, avoids prolonged medication, and may confer some long-term benefit.
\end{abstract}

Although the cornea is classically described as possessing immunological privilege, the protection this affords is only relative and rejection is still the commonest cause of corneal graft failure. ${ }^{1-3}$ Consequently immunosuppression is still routinely used in keratoplasty. In the majority of grafts topical corticosteroids provide sufficient immunosuppression, but in high-risk grafts other therapeutic agents may be required. At present the term 'high-risk cornea' encompasses a wide range of corneas at

From: Department of Ophthalmology, Groote Schuur Hospital and University of Cape Town, Cape Town, South Africa.

Correspondence to: Dr John C. Hill, Department of Ophthalmology, Medical School, University of Cape Town, Observatory 7925, Cape Town, South Africa. Fax: 021-4481145. different degrees of risk, and no universally accepted definition of high risk exists. Without such a definition it is difficult to compare and devise treatment regimens.

In this paper the definition of 'high risk' is discussed and a new classification is proposed. Immunosuppressive regimens, and other measures that are used to modify the immune response postoperatively, are reviewed and related to this new classification. Finally an outline of the methods used to manage corneal allograft rejection is presented.

\section{PROPHYLACTIC IMMUNOSUPPRESSION}

Virtually all corneal grafts receive immunosuppressive treatment post-operatively; in the vast majority this includes topical corticosteroid drops, which for many grafts is the only form of treatment given. The usual topical preparations used are $1 \%$ prednisolone and $0.1 \%$ dexamethasone, although weaker preparations such as $0.25 \%$ or $0.1 \%$ fluorometholone are also used, especially when the side-effects of topical corticosteroids need to be avoided. In a survey of Castroviego Cornea Society members, ${ }^{4}$ there was some agreement amongst respondents concerning the choice of preparation used post-operatively in corneal grafting, with $55-68 \%$ of respondents preferring $1 \%$ prednisolone acetate and a further 6-8 preferring $1 \%$ prednisolone without specifying the type. Although there was a certain degree of unanimity concerning the preparations used, there was a wide variation in the frequency of usage. In an avascular cornea undergoing a graft for the first time, $100 \%$ of respondents used topical steroids postoperatively; however, the frequency ranged from twice daily dexamethasone ointment to hourly $1 \%$ prednisolone acetate drops (including night-time!). The average frequency was four times daily, with $43 \%$ of respondents also using subconjunctival steroids and 7\% systemic steroids. In high-risk corneas the frequency range was the same, but the average frequency increased to seven times daily and 
$53 \%$ of respondents gave subconjunctival steroids and $23 \%$ gave systemic steroids. This surprisingly wide variation in treatment preference probably stems from a paucity of information concerning the optimal preparation, dosage, route of administration and duration of treatment.

The importance of post-operative immunosuppressive regimens on the incidence of graft failure is underscored by the findings of the Collaborative Corneal Transplantation Study (CCTS); $;^{5}$ the authors attributed the improved graft survival found in their high-risk cases to the use of intensive topical steroid therapy post-operatively, in addition to close personal follow-up, and excellent patient compliance and understanding. Although doubts have been raised as to whether all the patients in the CCTS were truly at high risk, there is general agreement that high-risk cases need to be more intensively treated postoperatively. This is confirmed by the respondents of the Castroviejo Cornea Society survey, ${ }^{4} 85 \%$ of whom modified their treatment regimen for patients at high risk of allograft rejection. In aphakic patients without steroid-induced glaucoma it has been suggested that long-term topical steroids be used on a daily basis for patients with vascularisation or other risk factors. ${ }^{6}$ Alkaline-damaged corneas and other high-risk patients may require higher maintenance doses of topical steroids. ${ }^{7,8}$

Although the term 'high risk' is frequently applied to grafts known to have an increased likelihood of graft rejection, there is not a universally accepted definition of a high-risk cornea. Many risk factors for graft failure are known, but the usual risk factors used to define high risk are those that predispose to graft rejection, and include recipient vascularisation, previous graft failure, and the aetiology of the original corneal disease. However, the individual importance of these risk factors and their relative importance to other risk factors has not been fully established. It has been suggested that any cornea being grafted for a disease other than keratoconus or one of the stromal dystrophies can be regarded as high risk, ${ }^{9}$ although most authors apply stricter criteria. In a study on cross-matching Stark et al. ${ }^{10}$ defined high risk as significant stromal vascularisation in at least three quadrants, extending into the visual axis. Foulks et al. ${ }^{11-13}$ used a similar definition, namely significant vascularisation of two or more quadrants of the corneal stroma into the optical zone or a history of an irreversible corneal allograft rejection. A similar definition was used by Belin et al. ${ }^{14}$ although they specified deep stromal vascularisation in two or more quadrants and added a cornea scarred by severe alkaline burns as a factor for defining high risk. Recently the CCTS ${ }^{15}$ and the topical cyclosporine study have used similar definitions of high risk, namely two or more quadrants of corneal stromal vascularisation, extending at least $2 \mathrm{~mm}$ into the cornea, or a previous graft rejection in the affected eye. In all these studies the degree of vascularisation was defined as the number of quadrants of vascularisation rather than the total number of vessels. It is therefore possible to define a cornea as being high risk when only two vessels are present, provided they are in different quadrants. Fine and Stein ${ }^{16}$ defined a cornea as being vascularised if only one vessel was present, and were able to demonstrate a higher risk of rejection in these cases. Khodadoust ${ }^{2}$ classified the degree of vascularisation into: avascular, mild (1-3 vessels), moderate (4-10 vessels) and heavy (>10 vessels). He found the incidence of rejection increased with the degree of vascularisation. In the heavily vascularised group, $65 \%$ of grafts started to reject and all succumbed despite treatment. Gibbs et al. ${ }^{17}$ likewise found an increased incidence of rejection with increased vascularisation.

The term high-risk cornea can therefore encompass corneas with as few as one or two vessels to corneas heavily vascularised in all four quadrants, such as those seen in severe alkali burns. This definition is too broad to act as a basis for selecting treatment regimes or to give patients requiring surgery an accurate prognosis.

The presence of a previously rejected graft has also been used as the sole criterion for defining high risk. However, it has been suggested that a previous graft failure from rejection is not itself a risk factor, ${ }^{18,19}$ but that the higher incidence of rejection results from the vascularisation occurring in the rejection process. In a paper ${ }^{20}$ reviewing the effect of some of the preoperative risk factors on graft survival, we have also shown that a previously rejected graft is not itself a risk factor. In avascular corneas, survival of a repeat graft was not significantly different from first-time grafts; but in vascular corneas repeat grafts did have a significantly worse survival. This would indicate that in avascular corneas, a history of a previously rejected graft should not be used as the sole criterion for defining high risk. In the same paper we showed that, using multivariate analysis, the only significant risk factor was the number of vascularised quadrants and not the total number of stromal vessels. Statistically there was a natural grouping of patients into the following groups: avascular corneas, corneas with 1 or 2 quadrants of vascularisation and corneas with $3+$ quadrants of vascularisation. These can be termed low-, medium- and high-risk corneas respectively.

Using this classification, post-operative prophylactic immunosuppressive regimens can be devised according to the -degree of risk. In low-risk (avascular) corneas, topical corticosteroids are probably sufficient although many surgeons would 
prefer to give a subconjunctival corticosteroid injection at the completion of surgery. A frequency of four times daily is probably adequate initially and can be tailed-off over a period of 4-6 months provided the eye is quiet. A rejection episode is most likely to occur within the first year ${ }^{1,12,21,22}$ and especially within the first 6 months. ${ }^{2,23,24}$ In a study of 37 high-risk keratoplasty patients $(3+$ quadrants of vascularisation) given only topical steroids, ${ }^{25}$ we found that 27 grafts $(62.2 \%)$ were lost from rejection at a mean time of 11.2 months, and a median time of 8 months, after operation. Of the 23 rejected grafts, 11 rejected during the first 6 months and 16 during the first year. Greater vigilance should be exercised in the early post-operative period, although the possibility of rejection is always present. In our study of high-risk grafts, ${ }^{25}$ it is apparent that topical steroids alone were not effective in preventing failure from rejection, although the dose of topical corticosteroids was arguably low (four times daily, tailing off to stop at 6 months unless there was persistent inflammation). In the same study ${ }^{25}$ a similar high-risk group was given oral prednisone ( $25 \mathrm{mg}$ daily for a month followed by $10 \mathrm{mg}$ daily for 3 months) in addition to topical steroids, but this did not confer any additional benefit. Medium- and high-risk cases should be given topical corticosteroids more frequently and for a longer duration, and most surgeons give a subconjunctival injection of corticosteroids at the time of operation. In the CCTS, high-risk grafts were given topical prednisolone acetate 2 hourly initially with dexamethasone ointment at night. The frequency and strength of the topical steroid was reduced until at 7 months only fluoromethalone once daily was used.

Although the increased frequency of topical steroids may be sufficient to reduce the incidence of rejection in the medium-risk patients, maximal doses of corticosteroids do not reliably prevent rejection in the high-risk cases. The addition of systemic steroids does not seem to confer any additional benefit. ${ }^{25}$ What other methods of modifying the immune response can be used? The role of histocompatibility matching in high-risk keratoplasty has not been resolved. Studies have shown improved graft survival using negative crossmatched grafts ${ }^{10,26}$ and histocompatibility matching between recipient and donor. ${ }^{12,13,17,19,22,27,28}$ The CCTS ${ }^{5}$ failed to show any significant benefit from either of these two approaches to tissue matching, although some benefit was demonstrated with ABO matching. Similarly, studies have demonstrated the benefit of topical cyclosporine (CSA) on graft failure in high-risk keratoplasty, ${ }^{14,29}$ but again a multicentre trial based on a protocol similar to the CCTS failed to find any benefit of topical CSA (personal communication). Both the CCTS and the topical CSA trial have been criticised for including patients who may not have been truly at high risk, and also the intensive topical corticosteroid regimen may have masked any beneficial effect from these two treatment modalities. Possibly with the newer methods of DNA tissue typing and greater understanding of the role of minor histocompatibility antigens, improved techniques will lead to histocompatibility matching becoming a viable treatment modality. The formulation of an effective vehicle for topical CSA and a more frequent usage than the twice daily regimen used in the topical CSA trial, may eventually prove the efficacy of this treatment modality. It is possible that we will need to look at multiple treatment regimens. In an animal high-risk model, ${ }^{30}$ we have shown that in vascularised corneas topical CSA significantly improved graft survival compared with untreated grafts. But it was grafts with a relatively good donor/ recipient histocompatibility match that survived whereas the poorly matched donor/recipient grafts rejected despite topical CSA. In our medium- and high-risk cases, to achieve high success rates we may need to consider multiple treatment modalities such as tissue matching in addition to treatment with both corticosteroids and CSA given topically.

One form of treatment that does effectively improve graft survival is systemic CSA. This is widely used in solid organ transplantation, but is rarely used in corneal transplantation because of the potential side-effects and cost of treatment. A number of reports have demonstrated the effectiveness of systemic CSA in preventing corneal graft failure from rejection in humans. ${ }^{25,31-33}$ In our early study $^{25}$ we compared the results of patients given topical corticosteroids and systemic CSA with those of patients given topical corticosteroids either alone or in combination with systemic steroids. There was a highly significant improvement in graft survival in patients given CSA compared with the other two groups $(p<0.0001)$. Initially CSA was given for 12 months, but because of the good results obtained we reduced the treatment time to 4 months with no apparent decrease in graft survival. A subsequent paper $^{33}$ showed that although a 4-6 month course of treatment significantly improved graft survival, a longer 12 month course of CSA resulted in better graft survival. The dosage of CSA was adjusted to keep blood levels within the therapeutic range, and preferably in the lower part of the range. The usual oral dosage of CSA to achieve this was 3-4 mg/kg per day. Systemic CSA appears to improve graft survival in our patients by both reducing the incidence of allograft rejection and increasing the number of rejection episodes that are reversed. ${ }^{34}$ These benefits of CSA therapy appear to persist after CSA is stopped, indicating that possibly some degree of immune privilege is re-established. Although 
patients with other organ transplants are given CSA indefinitely, it would appear that in corneal grafting CSA can be stopped after a period of time, although the optimal duration of treatment has not been established. We have reserved systemic CSA for high-risk patients (3+ quadrants of vascularisation) who have either bilateral disease or an affected only eye. A low incidence of side-effects was found, probably because our patients were generally systemically healthy. Any side-effects encountered tended to occur early, within a month or two of starting treatment, and the longer 12 month course was not associated with a higher complication rate. In patients who are blind from their corneal disease it is reasonable to use systemic CSA to restore vision; in patients with unilateral disease and a normal contralateral eye the indications for this potent drug are less clear.

\section{TREATMENT OF CORNEAL GRAFT REJECTION}

One important aspect of managing corneal graft rejection is patient education and awareness; early diagnosis and treatment can substantially improve the prognosis of graft rejection. The eye is exquisitely sensitive to changes in function that result in deteriorating vision. These should be easily detected by the patient and early help sought. Patients should appreciate that rejection can occur at any time after the first 10 days. with cases being reported 20 years ${ }^{35}$ and 31 years after surgery. ${ }^{24}$ The CCTS suggested that the single most important factor in graft survival was early detection and treatment of rejection episodes. A rejection episode involving the endothelial layer results in large numbers of endothelial cells being destroyed, ${ }^{36}$ and even if the rejection process is reversed sufficient cells may not survive to maintain graft clarity. ${ }^{7}$ Rejection episodes should therefore be reversed as quickly as possible to preserve the maximal number of endothelial cells. ${ }^{37}$ Patients should be particularly aware in the early postoperative period, during which time the eye is still irritable and the vision poor. In grafts with a good prognosis the incidence of rejection has been reported to be between $2.3 \%$ and $35 \%,{ }^{38-40}$ compared with high-risk grafts in which the incidence is between $40 \%$ and $68 \% .^{39.41 .42}$

A rejection episode can involve either a single layer or more than one layer of the cornea. Involvement in each layer exhibits a distinct clinical picture resembling the experimental findings of Khodadoust and Silverstein. $2,24,38,39,43$ Four types of corneal rejection have been described: epithelial rejection, subepithelial infiltrates, stromal, and endothelial rejection, ${ }^{24,43,44}$ but usually more than one type of graft rejection coexists. Endothelial rejection is the most severe form of corneal graft rejection, because this layer has no regenerative capabilities and significant cell loss can lead to chronic graft oedema. Two forms of endothelial rejection are described. The first is an advancing rejection, or Khodadoust line. This usually originates at a vascularised area of the cornea ${ }^{39}$ or at the site of an anterior synechia. ${ }^{45}$ Symptoms of rejection include pain. redness and loss of vision. Arentsen ${ }^{1}$ found that $45 \%$ of his patients with endothelial rejection had a Khodadoust line. In the other type of endothelial rejection, diffuse keratic precipitates are scattered across the donor endothelium although often they are not readily visible. The resultant diffuse damage to the endothelium in turn leads to diffuse graft oedema. ${ }^{2,24,39}$ It is not known what factors are responsible for the two types of rejection. It has been suggested that the vascularisation associated with the rejection line acts as a route for the afferent and efferent arcs of the rejection process. In the diffuse type it is suggested that the immunological route is via the anterior chamber. Thus differing routes of immunological contact may be responsible for the differences seen clinically. ${ }^{6}$ The reported incidence of endothelial rejection varies, partly because the studies used different post-operative immunosuppressant regimens and also because the study groups contained different proportions of high-risk cases.

It is often difficult to differentiate the graft oedema associated with rejection from other causes of endothelial cell loss, especially in the absence of other signs of rejection such as inflammation or the typical Khodadoust line. This can be particularly important in herpes simplex keratitis, a common reason for keratoplasty. In disciform keratitis there is inflammation, with graft oedema and keratic precipitates. In herpetic uveitis endothelial dysfunction is common and leads to graft oedema. If there is any doubt then antiviral medication should be given in addition to immunosuppressant therapy when there is a past history of herpes simplex infection. ${ }^{46}$ Because of the difficulties sometimes encountered in the diagnosis of rejection, Stulting et al. ${ }^{47}$ have suggested a terminology for rejection episodes. A definite rejection episode is defined as one producing corneal oedema and an endothelial rejection line, in a previously clear graft. A probable rejection episode is one with corneal oedema and inflammation (stromal infiltrate, keratic precipitates, cells in the anterior chamber, or ciliary injection) without a rejection line in a previously clear graft. A possible rejection episode is one with diffuse oedema (often occurring gradually) with no signs of inflammation or other diagnosable cause. If a graft never became clear after operation it should be regarded as a primary failure.

Corticosteroids, administered locally and systemi- 
cally, remain the mainstay in the treatment of graft rejection. Epithelial, subepithelial or stromal rejection are not usually associated with graft failure unless there is associated endothelial involvement, although stromal scarring with some loss of vision can occur. ${ }^{24}$ These forms of rejection do, however, indicate that recipient sensitisation has occurred and may be harbingers of impending endothelial rejection. Therefore, all episodes of rejection should be treated vigorously as early as possible.

In the survey of the members of the Castroviego Corneal Society mentioned earlier, ${ }^{4}$ the treatment preferences of surgeons were sought. For subepithelial infiltrates and epithelial rejection $93 \%$ of surgeons gave topical steroids (dosage range 0 to 24 drops per day), approximately $10 \%$ gave subconjunctival steroids and approximately $10 \%$ gave systemic steroids. For endothelial rejection $100 \%$ gave topical steroids (dosage range 4 to 24 drops per day), approximately $55 \%$ gave subconjunctival steroids and approximately $35 \%$ gave systemic steroids. It is apparent that the intensity of treatment given for a rejection episode varies tremendously between surgeons. The following regimen has been suggested by Wilson and Kaufman, ${ }^{6}$ and is similar to regimens advocated by other studies. ${ }^{7,24,35}$ For non-endothelial types of rejection topical $1 \%$ prednisolone acetate or $0.1 \%$ dexamethasone sodium phosphate drops are given six times daily for approximately a week and then tapered of over a period of 3-5 weeks. For endothelial rejection the drops are given hourly during the waking hours with dexamethasone sodium phosphate $0.05 \%$ or betamethasone $0.1 \%$ ointment at night. In addition a subconjunctival injection of 2 $\mathrm{mg}$ dexamethasone sodium phosphate is given. The drops are administered hourly for 3 days, then 2 hourly for several days and then slowly tapered off over several weeks or months. The ointment is usually stopped after 2 or 3 weeks. If there is minimal response to treatment a repeat subconjunctival injection can be given after 2 or 3 days. As an alternative to subconjunctival injections, collagen shields in conjunction with corticosteriod drops have been suggested, the shield acting as a reservoir for the drug. 48

In severe rejection episodes involving the endothelium some authors use only topical corticosteroid drops, ${ }^{6,18,46}$ others treat with the addition of systemic steroids. $^{1,7,24}$ In a recent study Boisjoly et al. ${ }^{18}$ reported that 17 of 23 grafts $(73.9 \%)$ that developed a single episode of endothelial rejection failed when only topical corticosteroids were used. We have also found that the use of topical corticosteroids alone, in cases of definite and probable rejection, has a low success rate $(39 \%)$ in reversing graft rejection (unpublished data). Because of the lower success rates in endothelial rejection many authors prefer to give systemic corticosteroids to these cases provided that there are no contraindications. The usual dose is $60-80 \mathrm{mg}$ daily (depending on body weight) for 3 days, tapering off over a week or two. A single intravenous dose of $125 \mathrm{mg}$ methylprednisolone sodium has also been advocated in the management of severe graft rejection. ${ }^{49}$

We now prefer to use a single $500 \mathrm{mg}$ intravenous pulse of methylprednisolone instead of oral steroids for the treatment of severe endothelial rejection..$^{50,51}$ This form of corticosteroid administration appears to be at least as effective as oral administration and avoids the potential side-effects of prolonged oral medication. Our studies showed that patients given a single $500 \mathrm{mg}$ intravenous pulse of methylprednisolone (pulse group) fared at least as well as patients given $60-80 \mathrm{mg}$ prednisone by mouth tapered off over 2 weeks (oral group); all patients also received hourly topical corticosteroid drops. In the pulse group $79.2 \%$ of grafts survived compared with $62.5 \%$ in the oral group $(p=0.17)$. In this study the interval between onset of symptoms of rejection and treatment ranged from 3 to 21 days (median 8 days). However, if we considered patients who presented early ( 8 days or less) then $92.8 \%$ of grafts in the pulse group recovered compared with $54.5 \%$ in the oral group $(p<0.05)$. This finding underscores the importance of early diagnosis and treatment in graft rejection. Another interesting finding was the fate of the grafts that recovered; only $25 \%$ of those in the pulse group had a further rejection episode compared with $66.7 \%$ in the oral group $(p<0.025)$. This suggests that pulse therapy may confer some degree of long-term protection. Further studies in which a second pulse was given either 24 or 48 hours after the first pulse, showed no improvement in graft survival. ${ }^{52}$ The mechanism by which pulse therapy works is not known but it appears able to 'reset' an aberrant immune response by the simultaneous occurrence of inhibition of the proliferating clone, the temporary removal of recirculating $\mathrm{T}$ lymphocytes from the blood and eye, and the profound suppression of peripheral inflammation. ${ }^{53}$ This immunological manipulation has been shown to induce long-term remissions in destructive corneal and scleral disease. ${ }^{53}$ A similar explanation probably accounts for the decreased incidence of subsequent rejection episodes found in the patients treated with pulse therapy in our study.

In the past other immunosuppressant agents such as azathioprine (Imuran) ${ }^{54-59}$ have been used to help prevent rejection and to treat rejection episodes when they occur. But the results show little advantage over corticosteroids and their use requires great care because of potentially serious side-effects.

Regimens for reversing graft rejection have had 
variable success rates, with reversal rates of $50-91 \%$ being reported. This variation results from the studies reporting rejection reactions of different severity, using different routes of corticosteroid therapy depending on the severity of the immune reaction, different degrees of corneal vascularisation and other risk factors, and variable delays in presentation and treatment. ${ }^{2,11,16,17,24,38,60,61}$ Fine and Stein $^{16}$ found that in avascular corneas the reversal rate was $67 \%$, but that in vascularised corneas only $50 \%$ of rejection episodes were successfully reversed. It would appear that in highrisk cases rejection is more likely to occur and is also more difficult to reverse. This emphasises the necessity of having accurate criteria for defining high risk in order that prophylactic and therapeutic regimens for treating rejection can be devised. A universally accepted definition of high risk would allow the treatment regimens used in different centres to be compared. The final goal would be to determine the optimal dosage, frequency and duration of the immunosuppressant agents that are used post-operatively in keratoplasty and in the treatment of corneal graft rejection.

This study was partially funded by a grant from the South African Medical Research Council.

Key words: Allograft rejection, Corneal graft, Corticosteroids, Cyclosporine, Immunosuppression, Keratoplasty.

\section{REFERENCES}

1. Arentsen JJ. Corneal transplant allograft reaction: possible predisposing factors. Trans Am Ophthalmol Soc $1983 ; 81: 361-402$.

2. Khodadoust AA. The allograft rejection reaction: the leading cause of late graft failure of clinical corneal grafts. In: Porter R, Knight J, editors. Corneal graft failure. Ciba Foundation Symposium 15. Amsterdam: Elsevier, 1973:151-64.

3. Coster DJ. Some factors which affect the visual outcome of corneal transplantation. Eye 1991;5:265-78.

4. Rinne JR, Stulting RD. Current practices in the prevention and treatment of corneal graft rejection. Cornea 1992;11:326-8.

5. The Collaborative Corneal Transplantation Studies Research Group. The Collaborative Corneal Transplantation Studies (CCTS). Effectiveness of histocompatibility matching in high-risk corneal transplantation. Arch Ophthalmol 1992;110:1392-403.

6. Wilson SE, Kaufman HE. Graft failure after penetrating keratoplasty. Surv Ophthalmol 1990;34:325-56.

7. Smolin G, Goodman D. Corneal graft reaction. Int Ophthalmol Clin 1988;28:30-6.

8. Brown SI, Tragakis MP, Pearce DB. Corneal transplantation for severe alkali burns. Trans Am Acad Ophthalmol Otolaryngol 1972;76:1266-74.

9. Coster D, Williams K. Surgical manoeuvres to reduce the impact of corneal graft rejection. Dev Ophthalmol 1989;18:156-64.

10. Stark WJ, Taylor HR, Bias WB, Maumenee AE. Histocompatibility (HLA) antigens and keratoplasty. Am J Ophthalmol 1978;86:595-604.

11. Foulks GN, Sanfilippo F. Beneficial effects of histo- compatibility in high-risk corneal transplantation. Am J Ophthalmol 1982;94:622-9.

12. Foulks GN, Sanfilippo FP, Locascio JA, et al. Histocompatibility testing for keratoplasty in high-risk patients. Ophthalmology 1983;90:239-44.

13. Sanfilippo F, MacQueen JM, Vaughn WK, Foulks GN. Reduced graft rejection with good HLA A and B matching in high risk corneal transplantation. $\mathrm{N}$ Engl J Med 1986;315:29-35.

14. Belin MW, Bouchard CS, Frantz S, Chmielinska J. Topical cyclosporine in high-risk corneal transplants. Ophthalmology 1989;96:1144-50.

15. The Collaborative Corneal Transplantation Studies Research Group. Design and methods of the Collaborative Corneal Transplantation Studies. Cornea 1993; 12:93-103.

16. Fine $M$, Stein $M$. The role of corneal vascularisation in human corneal graft reactions. In: Porter R, Knight K, editors. Corneal graft failure. Ciba Foundation Symposium 15. Amsterdam: Elsevier, 1973:193-204.

17. Gibbs DC, Batchelor JR, Werb A, et al. The influence of tissue-type compatibility on the fate of full thickness corneal grafts. Trans Ophthalmol Soc UK 1974; 94:101-26.

18. Boisjoly HM, Bernard P, Dube I, et al. Effect of factors unrelated to tissue matching on corneal transplant endothelial rejection. Am J Ophthalmol 1989; 107:647-54.

19. Völker-Dieben HJM, D’Amaro J, Kok-van Alphen CC. Hierarchy of prognostic factors for corneal allograft survival. Aust NZ J Ophthalmol 1987;15:11-8.

20. Hill J, Lombard C. The relative importance of risk factors used to define high-risk. Submitted for publication.

21. Meyer RF. Corneal allograft rejection in bilateral penetrating keratoplasty: clinical and laboratory studies. Trans Am Ophthalmol Soc 1986;84:664-742.

22. Boisjoly HM, Roy R, Dubé I, et al. HLA-A, B and DR matching in corneal transplantation. Ophthalmology 1986;93:1290-7.

23. Stark WJ, Opelz G, Newsome D, et al. Sensitisation to human lymphocyte antigens by corneal transplantation. Invest Ophthalml Vis Sci 1973;12:639-45.

24. Alldredge OC, Krachmer JH. Clinical types of corneal rejection: their manifestations, frequency, preoperative correlates, and treatment. Arch Ophthalmol 1981; 99:599-604.

25. Hill JC. The use of cyclosporine in high-risk keratoplasty. Am J Ophthalmol 1989;107:506-10.

26. Stark WJ, Taylor HR, Datiles M, et al. Transplantation antigens and keratoplasty. Aust J Ophthalmol 1983; 11:333-9.

27. Batchelor JR, Casey TA, Gibbs DC, et al. HLA matching and corneal grafting. Lancet 1976;1:551-4.

28. Özdemir Ö. A prospective study of histocompatibality testing for keratoplasty in high-risk patients. $\mathrm{Br} \mathrm{J}$ Ophthalmol 1986;70:183-6.

29. Goichot-Bonnat L, Chemla P, Pouliquen Y. Cyclosporine A collyre dans la prévention du rejet de greffe de cornée à haut risque. II. Résultats cliniques postopératoires. J Fr Ophtalmol 1987;10:213-7.

30. Maske R, Hill J, Horak S. Mixed lymphocyte culture responses in rabbits undergoing corneal grafting and topical cyclosporine treatment. Cornea 1994;13:324-30.

31. Hill JC. The use of systemic cyclosporin in human corneal transplantation: a preliminary report. Doc Ophthalmol 1986;62:337-44.

32. Miller K, Huber C, Niederwieser D, Göttinger W. Successful engraftment of high risk corneal allografts 
with short term immunosuppression with cyclosporine. Transplantation 1988;45:651-3.

33. Hill J. Systemic cyclosporine in high-risk keratoplasty: short versus long term therapy. Ophthalmology 1994;101:128-33.

34. Hill J. Systemic cyclosporine in high-risk keratoplasty: long term results. Eye 1995;9: in press.

35. Chandler JW. Immunologic considerations in corneal transplantation. In: Kaufman $\mathrm{H}$, McDonald MB, Barron BA, Waltman SR, editors. The cornea. New York: Churchill Livingstone, 1988:725-41.

36. Watson, AP, Simcock PR, Ridgway AEA. Endothelial cell loss due to repeated traumatic wound dehiscence after penetrating keratoplasty. Cornea 1987;6:216-8.

37. Brooks AMV, Grant G, Gillies WE. Assessment of the corneal endothelium following keratoplasty. Aust NZ J Ophthalmol 1989;17:379-85.

38. Chandler JW, Kaufman HE. Graft reactions after keratoplasty for keratoconus. Am J Ophthalmol 1974;77:543-7.

39. Khodadoust AA, Silverstein AM. Transplantation and rejection of individual cell layers of the cornea. Invest Ophthalmol Vis Sci 1969;8:180-95.

40. Donshik PC, Cavanagh HD, Boruchoff SA, Dohlman $\mathrm{CH}$. Effect of bilateral and unilateral grafts on the incidence of rejections in keratoconus. Am J Ophthalmol 1979;87:823-6.

41. Maumenee AE. The influence of donor-recipient sensitisation on corneal grafts. Am J Ophthalmol 1951;34:142-52.

42. Ehlers N, Olsen T, Johnson HE. Corneal graft rejection probably mediated by antibodies. Acta Ophthalmol (Copenh) 1981;59:119-25.

43. Stark WJ. Transplantation immunology of penetrating keratoplasty. Trans Am Ophthalmol Soc 1980; 78:1079-117.

44. Maumenee AE. Clinical patterns of corneal graft failure. In: Porter R, Knight J, editors. Corneal graft failure. Ciba Foundation Symposium 15. Amsterdam: Elsevier, 1973:5-15.

45. Smolin G, Biswell R. Corneal graft rejection associated with anterior iris adhesion: case report. Ann Ophthalmol 1978;10:1603-4.

46. Ficker LA, Kirkness CM, Rice NSC, Steele ADMG. The changing management and improved prognosis for corneal grafting in herpes simplex keratitis. Ophthalmology 1989;96:1587-96.

47. Stulting RD, Waring GO, Bridges WZ, Cavanagh HD. Effect of donor epithelium on corneal transplant survival. Ophthalmology 1988;95:803-12.

48. Hwang DG, Stern WH, Hwang PH, MacGowan-Smith LA. Collagen shield enhancement of topical dexamethasone penetration. Arch Ophthalmol 1989; 107:1375-80.

49. Braude LS, Chandler JW. Corneal allograft rejection: the role of the major histocompatibility complex. Surv Ophthalmol 1983;27:290-305.

50. Hill JC, Maske RM, Watson P. Corticosteroids in corneal graft rejection: oral versus single pulse therapy. Ophthalmology 1991;98:329-33.

51. Hill JC, Maske R, Watson PG. The use of a single pulse of intravenous methylprednisolone in the treatment of corneal graft rejection: a preliminary report. Eye 1991;5:420-4.

52. Hill J, Ivey A. Corticosteroids in corneal graft rejection: double versus single pulse therapy. Cornea 1994; 13:383-8.

53. Meyer PA, Watson PG, Franks W, Dubord P. 'Pulsed' immunosuppressive therapy in the treatment of immunologically induced corneal and scleral disease. Eye 1987;1:487-95.

54. Hughes WF, Kallmeyer J. Aetiology and treatment of the corneal homograft reaction including azathioprine (imuran). S Afr Med J 1967;41:548-51.

55. Mackay IR, Bigwell JL, Smith PH, Crawford BA. Prevention of corneal-graft failure with the immunosuppressive drug azathioprine. Lancet 1967;2:479-82.

56. Polack FM. Effect of azathioprine (imuran) on corneal graft reaction. Am J Ophthalmol 1967;64:233-44.

57. McCulloch C. Immunosuppression and keratoplasty. Can J Ophthalmol 1971;6:161-9.

58. Philipsen WMJG. The treatment of corneal transplantations with Imuran. Ophthalmologica 1972; 165:529-32.

59. Barraquer J. Immunosuppressive agents in penetrating keratoplasty. Am J Ophthalmol 1985;100:61-4.

60. Hughes WF. Keratoplasty for corneal dystrophies. Am J Ophthalmol 1960;50:1100-14.

61. Williams KA, Sawyer MA, White MA, et al. Report from the Australian Corneal Graft Registry. Transplant Proc 1989;21:3142-4. 